\title{
29GHz-bandwidth monolithically integrated EAM-VCSEL
}

\author{
Ludovic Marigo-Lombart ${ }^{1, *}$, Christophe Viallon ${ }^{1}$, Alexandre Rumeau ${ }^{1}$, Alexandre Arnoult ${ }^{1}$, Stéphane \\ Calvez $^{1}$, Antoine Monmayrant ${ }^{1}$, Olivier Gauthier-Lafaye ${ }^{1}$, Hugo Thienpont ${ }^{2}$, Krassimir Panajotov ${ }^{2}$, \\ Guilhem Almuneau ${ }^{1}$ \\ 1. LAAS-CNRS, Université de Toulouse, CNRS, 31031 Toulouse, France
}

2. Department of Applied Physics and Photonics, B-Phot, Vrije Universiteit Brussel (VUB), Pleinlaan 2, B-1050 Brussels, Belgium

The increase in modulation bandwidth of a vertical-cavity surface-emitting laser (VCSEL) can be achieved through the vertical integration of a modulator onto the laser. This approach has already been proposed and demonstrated to be competitive [1-3]. In this specific case, a double mesa structure with three contacts is needed to apply a high-frequency voltage signal to the modulator section and inject a CW current into the underlying VCSEL section. The middle contact serves as a shared ground. Indeed, splitting the emitting and the modulating parts circumvents the carrier dynamics limitation of the modulation bandwidth encountered in direct currentmodulated VCSELs. However, the parasitic capacitances of the access line and contact pad play a significant role in the high-frequency limit of such a device.

In this paper, we present the design, fabrication and modulation characteristics of an electro-absorption modulator (EAM) vertically-integrated onto an 850-nm VCSEL that is compatible with high-frequency operation. The two optical cavities are such that their mutual optical coupling is small to improve the modulation properties at high frequencies. This EAM-VCSEL device has been successfully fabricated by molecular beam epitaxy, followed by an original technological process [4]. In particular, planarized BCB layers and microstrip access lines have been implemented to optimize the high-frequency signal injection. Thanks to the optimized wavelength detuning between the cavities resonances of the EAM and the VCSEL and respectively to the quantum well absorption tail of the modulator, the EAM modulator exhibits a modulation depth of more than $40 \%$ within a $4 \mathrm{~V}$ span. This vertically integrated EAM-VCSEL approach enables also to minimize the wavelength chirp during the modulation, which in our case is lower than $80 \mathrm{pm}$. Finally, 3-dB frequency bandwidths up to $29 \mathrm{GHz}$ were measured on both stand-alone vertical EAM devices and vertically-integrated EAM-VCSELs.

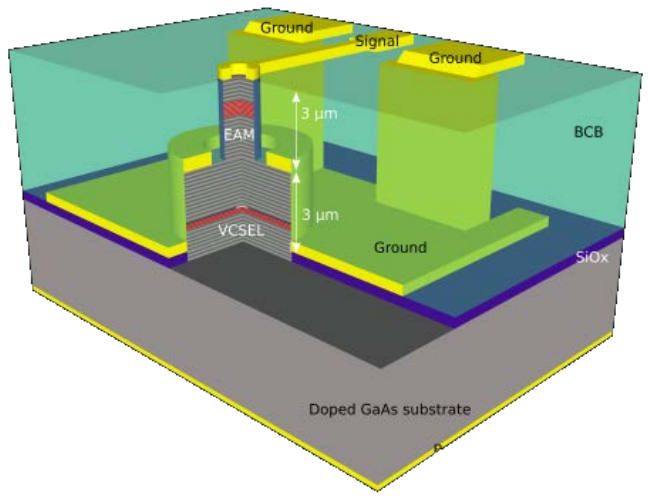

(a)

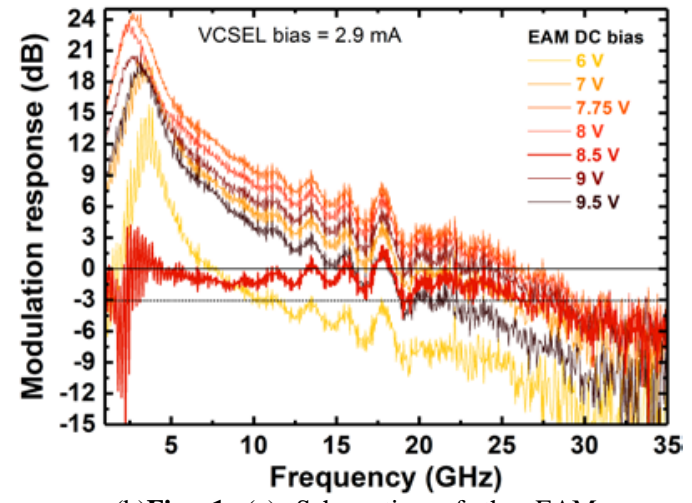

(b)Fig. 1 (a) Schematics of the EAM-

VCSEL structure under study with planarized BCB and (b) high-frequency modulation response of the EAM-VCSEL device with different DC bias voltages applied to the EAM.

\section{References}

[1] M. Zujewski, H. Thienpont, and K. Panajotov, “Electrical Design of High-Speed Electro-Optically Modulated Coupled-Cavity VCSELs,” J. Lightwave Technol. 29, 2992 (2011).

[2] T. D. Germann, W. Hofmann et al., "Electro-optical resonance modulation of vertical-cavity surface-emitting lasers,” Opt. Express 20, 5099 (2012).

[3] M. Yakimov, J. v. Eisden et al., "Concept of feedback-free high-frequency loss modulation in detuned duo-cavity vertical cavity surfaceemitting laser,” J. Vac. Sci. Technol. B 28, no. 3 (2010).

[4] L. Marigo-Lombart, A. Arnoult, L. Mazenq, P. Dubreuil, B., N. Mauran, H. Thienpont, K. Panajotov and G. Almuneau, "Single

lithography-step self-aligned fabrication process for Vertical-Cavity Surface-Emitting Lasers,” Mater. Sci. Semicond. Process. 61, 35 (2017). 